\title{
Cheekbone and cheek area augmentation with the Briseida technique: Analysis of 1,000 cases and 8 years of experience
}

\author{
Natalia Ribé \\ Instut Dra. Natalia Ribé, Barcelona, Spain
}

Email address:

nribe@institutnataliaribe.com

\section{To cite this article:}

Natalia Ribé. Cheekbone and Cheek Area Augmentation with the Briseida Technique: Analysis of 1,000 Cases and 8 Years of Experience. Journal of Surgery. Special Issue: Breakthroughs in Aesthetic Medicine. Vol. 3, No. 1-1, 2015, pp. 8-10. doi: 10.11648/j.js.s.2015030101.13

\begin{abstract}
Background. Non-surgical implant procedures have been used in facial rejuvenation, whether to improve the physical appearance or to correct asymmetries in the face. These procedures have quite a few advantages over surgical procedures, including an immediate cosmetic result and a short recovery period. Methods: The Briseida technique involves infiltrating a filler (Hyaluronic Acid) in a linear, retrograde and fan-shaped fashion at supraperiosteal or subcutaneous level. 1,000 patients between 25 and 75 years of age, treated during the past 10 years for cheekbone and/or cheek area augmentation with the Briseida technique, have been included in this revision. Results: shorter down-time, less product required, no serious adverse effects, longlasting results. Conclusions: The technique allows the oval of the face to be re-modelled. It is minimally invasive, quickly performed, and produces natural results with few and mild side effects.
\end{abstract}

Keywords: Hyaluronic Acid, Filler, Cheekbone, Cheek, NASHA

\section{Introduction}

A good balance in the contours of the face is closely connected to the spatial relationship existing between the cheekbones, the cheeks, and the oval of the face. It is this balance that can make a face more beautiful. The 'triangle of youth' treatment was specifically designed by our team in order to harmonize the appearance, improve face proportions, and rejuvenate. The key step of this process was the filling of the cheekbones, which is extremely helpful in the recovery of the facial contour. The aim of this work is to evaluate the impact of cheek area and cheekbone augmentation with Hyaluronic Acid and with a specially non-traumatic procedure: Briseida Technique.

In recent years, various non-surgical implant procedures have been used in facial rejuvenation, whether to improve the appearance or to correct asymmetries. These procedures have quite a few advantages over surgical procedures, including an immediate cosmetic result and a short recovery period [1].

The technique initially recommended for the implanting of the filler assessed in this review involved an incision in the skin and the use of a cannula. Later on, good results were demonstrated injecting the product with a needle, with many advantages over cannula injection [2], and that may even determine, up to some extent, the success of the whole procedure.

Non-Animal origin Stabilized Hyaluronic Acid (NASHA ${ }^{\circledR}$ ) is specially designed to add volume to certain areas of the face. The safety and durability of these implants, and their high level of tolerance have already been demonstrated in plenty of studies $[3,4,5,6,7,8]$.

\section{Material and Methods}

One thousand clinical records of patients between 25 and 75 years of age were included in this revision (69\% women, $31 \%$ men), all of them patients of the Institute Natalia Ribé, Barcelona, Spain. No further inclusion criteria were applied to the subjects.

This protocol conforms to the provisions of the Declaration of Helsinki of the World Medical Association for the respect of ethical principles in medical research involving human subjects.

The fillers used were: Restylane Sub-Q from 2004 to 
September 2011 (Q-Med AB, Seminariegatan 21, SE-752 28 Uppsala, Sweden) and Restylane Sub-Q lidocaine from October 2011 to 2012 (Q-Med AB, Seminariegatan 21, SE-752 28 Uppsala, Sweden). Restylane ${ }^{\circledR}$ Sub-Q is a gel composed of $20 \mathrm{mg} / \mathrm{ml}$ stabilized particles of Non-Animal origin Stabilized Hyaluronic Acid -NASHA®- $(1,000$ particles $/ \mathrm{ml})$. This resulted in smaller volumes of product required for the injection into the supra-periosteal and subcutaneous layers, and for the creation of more clearly-defined facial contours [3]. Restylane Sub-Q lidocaine is a gel composed of $20 \mathrm{mg} / \mathrm{ml}$ stabilized particles of Non-Animal origin Stabilized Hyaluronic Acid -NASHA®(1,000 particles/ml) and $3 \mathrm{mg} / \mathrm{ml}$ of lidocaine (no anaesthetic cream was used). Both compositions differ solely in the 3 $\mathrm{mg} / \mathrm{ml}$ of lidocaine.

The preparation prior to implanting included a standard clinical medical record, with a signed informed consent and photograph taking. The patient was evaluated, and the zone in which the implant was to be performed, and the dose. Tthe recommended maximum volume per injected area was: $2 \mathrm{ml}$ to $4 \mathrm{ml}$ per session. The area to be treated was then marked out, following the Hinderer model (Fig. 1) [9]. A topical anaesthetic cream was applied to the area to be treated (only when required), 30 to 60 minutes before performing the implant procedure.

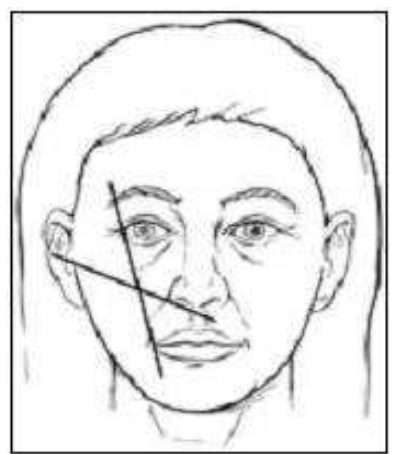

Fig 1. Pre treatment marking. The area to be treated was marked out, following the Hinderer model. The injection site can be find in the exact place where the black lines cross each other

The technique consisted in infiltrating the product to be implanted by means of a needle $23 \mathrm{G} / 30 \mathrm{~mm}$ in a linear, retrograde and fan-shaped fashion. After the anatomical identification of the areas to be treated, the needle was inserted through a single point (Fig. 2). The needle was placed perpendicular to the surface of the skin, in order to ensure the correct plane of injection and to minimize the risk of injury. Then, once the gel was being injected, the needle was simultaneously displaced to cover the whole injection area. It was important to stop pressing the piston of the syringe to make sure that the product was being deposited in the appropriate plane. In the cheekbone area, the injection was supra-periosteal, and the implant was deposited between the orbicular and zygomatic muscles and the periosteum. In the area of the cheeks, the injection was subcutaneous, deep, and over the muscle. The whole intervention took 15 to 20 minutes. Afterwards, a gentle massage was performed in order to ensure that the implant stayed in the correct place.

Depending on the case, the treatment was completed in one, two or three sessions. The sessions took place at 2 to 3 week intervals. The treatment took place in the medical office just like any other filling procedure, without stitches.

The post-treatment recommendations (in addition to those shown in the product technical data sheet) were: local application of ice, avoiding of extreme temperatures, gentle massaging of the treated area, sleeping in a supine position for the first few days after infiltration, and avoiding the use of a full-face helmet.

\section{Results}

The patients were able to resume their work and social lives immediately, with a normal facial appearance.

In almost all cases, the total amount of injected product was 2 to $6 \mathrm{ml}$. The areas which required a greater volume of product were the cheekbones and the cheeks, and those which required a smaller volume were the chin and the edge of the jaw. The patients for whom a greater volume of product was required were those who had lost a substantial amount of weight and those in whom two areas of the face were treated.

Other than the expected usual mild side effects, there were no serious or moderate adverse effects. Two special mild adverse effects were recorded: a) inflammation of the treated area, more specifically one cheek, which was related to a dental treatment in the days following the implant (requiring the patient to remain open-mouthed for a long period of time) and was resolved with oral anti-inflammatory drugs and topical treatment with corticosteroids for 48 hours; and b) a slight displacement of the product due to the use of a full-face helmet in the hours immediately following the treatment.

In cases where $1 \mathrm{ml}$ had been used in each side, the product was still palpable on examination after 9 months. In cases were $2 \mathrm{ml}$ had been injected into each side (cheekbones or cheeks), the implant lasted for an average of 12 months. In patients in whom $4 \mathrm{ml}$ had been injected into each side, the implant lasted for an average of 18 months.

\section{Discussion}

The Briseida technique for cheekbone implanting is minimally invasive, rapidly performed, and gives a final effect that looks natural. A good knowledge of the anatomy of the face and a great dexterity in manipulating the needle to perform the procedure are required.

The Briseida technique is the result of simplification to the greatest extent possible of the technique for placement of liquid implants into cheekbones and cheeks.

The effects obtained with the Briseida technique are natural, and patients can resume their social and working lives immediately after the intervention.

As regards material, I am a staunch defender of the needle as opposed to the cannula, because it provides much more control over the plane of injection and the quantity of product injected, and because minimal or no subsequent inflammatory 
reaction of the kind produced by the entry and exit of a cannula is present. $\mathrm{Wu}$ [2] reported an improvement in the precision of the implant and in the control over the volume injected. The patients feel more comfortable during the procedure than they do when the cannula is used, and express great satisfaction with this treatment. The technique which uses the needle is more comfortable for the patient, as shown by $\mathrm{Wu}$ et al [2] in a comparative study of the implant techniques with a cannula and with a needle.

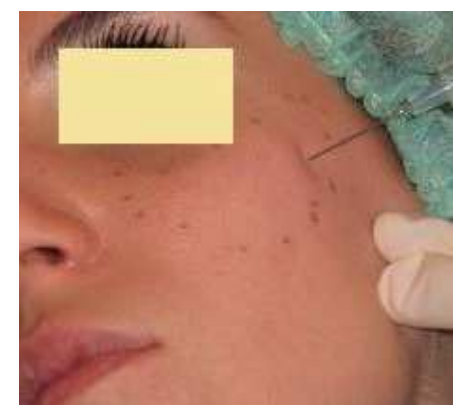

Fig 2. Injection site. Note: the needle is placed perpendicular to the surface of the skin, in order to ensure the correct plane of injection and to minimize the risk of injury.
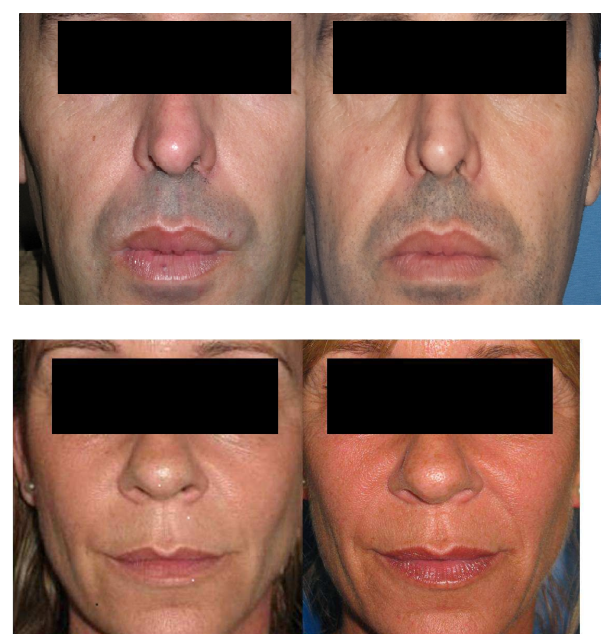

Fig 3. Two cases. Pre (left) and post (right) treatment picture of Hyaluronic Acid implantation with Briseida technique.

\section{Acknowledgments}

The author would like to thank her team and for their contribution to this work.

\section{References}

[1] Verpaele A., Strand A. Restylane SubQ, a non-animal stabilized hyaluronic acid gel for soft tissue augmentation of the mid- and lower face. Aesth Plast Surg 2006;26(Suppl):10-7.

[2] Wu W., Carlisle I., Huang P., Ribé N., Russo R., Schaar C., Verpaele A., Strand A. Novel administration technique for large-particle stabilized hyaluronic acid-based gel of nonanimal origin in facial tissue augmentation. Aesth Plast Surg 2010;34(1):88-95.

[3] DeLorenzi C., Weinberg M., Solish N., Swift A. The long-term efficacy and safety of a subcutaneously injected large-particle stabilized hyaluronic acid-based gel of nonanimal origin in esthetic facial contouring. Dermatol Surg 2009;35(Suppl 1):313-21.

[4] Bugge H, Negaard A, Skeie L, Bergersen B. Hyaluronic acid treatment of facial fat atrophy in HIV-positive patients. HIV Med 2007;8(8):475-82.

[5] Malhotra R. Deep orbital Sub-Q restylane (nonanimal stabilized hyaluronic acid) for orbital volume enhancement in sighted and anophthalmic orbits. Arch Ophthalmol 2007 Dec;125(12):1623-9.

[6] Lowe N.J., Grover R. Injectable hyaluronic acid implant for malar and mental enhancement. Dermatol Surg 2006; 32(7):881-5.

[7] Skeie L., Bugge H., Negaard A., Bergersen BM.Skeie L., Bugge H., Negaard A. y Bergersen BM. Large particle hyaluronic acid for the treatment of facial lipoatrophy in HIV-positive patients: 3-year follow-up study. HIV Med 2010;11(3):170-7.

[8] Naoum C. Cheek and facial contour augmentation with subcutaneous application of hyaluronic acid in HIV-positive patients. Kosmetische Medizin 2006;4:170-3.

[9] Hinderer U. Profileplasty. Int Macro. J Aesthic. Plast. Surg 1971;Sep:1-12. 